\title{
Lithium Depletion Boundary Ages of Young Stars: Inconsistencies in Pre-Main Sequence Models
}

\author{
Inseok Song ${ }^{1}$ \\ ${ }^{1}$ Department of Physics \& Astronomy, University of Georgia, Athens, GA 30602 \\ email: song@uga.edu
}

\begin{abstract}
For proper interpretations of various phenomena in young stars and planetary systems, knowledge of accurate stellar ages is very important. Among a handful of age dating methods commonly used for young $(\lesssim 500 \mathrm{Myr})$ stars, lithium depletion boundary (LDB) ages have recently become the most cited and accepted age estimates. However, because of inconsistencies in theoretical evolutionary models, especially for lithium depletion calculations, one has to be cautious in using LDB ages. For a given luminosity, the lithium depletion process is too slow, causing LDB ages to appear older. Various stellar processes affect the surface lithium abundance, and these effects include star spots, accretion history, and magnetic fields. Until we have a self-consistent theoretical evolutionary model for young stars including all relevant stellar effects, caution should be taken when LDB ages are used.
\end{abstract}

Keywords. stars: fundamental parameters (ages), stars: pre-main-sequence, stars: abundances

\section{Introduction}

Obtaining an accurate age estimate of a young star is important because ages provide the basis for proper interpretations of various aspects of the formation and evolution of young stars and planets. It is especially important to precisely age-date nearby young moving groups because their ages overlap with important planet formation epochs over the range of $10-100 \mathrm{Myr}$.

Ages of nearby young moving groups (NYMGs) can be estimated by comparing various activity indicators against patterns from the members of open clusters with well determined ages (see Zuckerman \& Song 2004). The Pleiades ( 100 Myr) and Hyades ( $\sim 650 \mathrm{Myr})$ clusters have been used as the best age anchors in such stellar age-dating. Ages of NYMGs younger than the Pleiades have been estimated using sparser clusters, such as IC 2602 and $\eta$ Cha, as age calibrators. Because this age-dating is essentially a relative age ordering, uncertainties in age dating are limited by the available age calibrators. Typical uncertainties in commonly used age determination methods for $\sim 10 \mathrm{Myr}$ old stars are ${ }_{-5}^{+10}$ Myr. Surface lithium abundance can be effectively used as a clock because Li burning (1) is fast, (2) starts at temperature of about 2 million Kelvin, and (3) is sensitive to the internal convective structure. At a given age beyond $\sim 8-10 \mathrm{Myr}$, the Li $\lambda 6708$ feature disappears among early M-type stars and suddenly reappears for types around mid-M; this is the so-called lithium depletion boundary (LDB). Because young stars around this LDB are fully convective, the precise location of the LDB in $T_{e f f}$ and luminosity can be readily transformed to a mass (hence an age) by using theoretical pre-main sequence (PMS) evolutionary models. LDB ages obtained for open clusters are typically $50 \%$ older than upper main sequence fitting ages, and this LDB-based age dating technique has been used for some NYMGs. The age of the $\beta$ Pictoris moving group (BPMG) has been estimated to be $\sim 12 \mathrm{Myr}$ based on various combinations of age-dating 


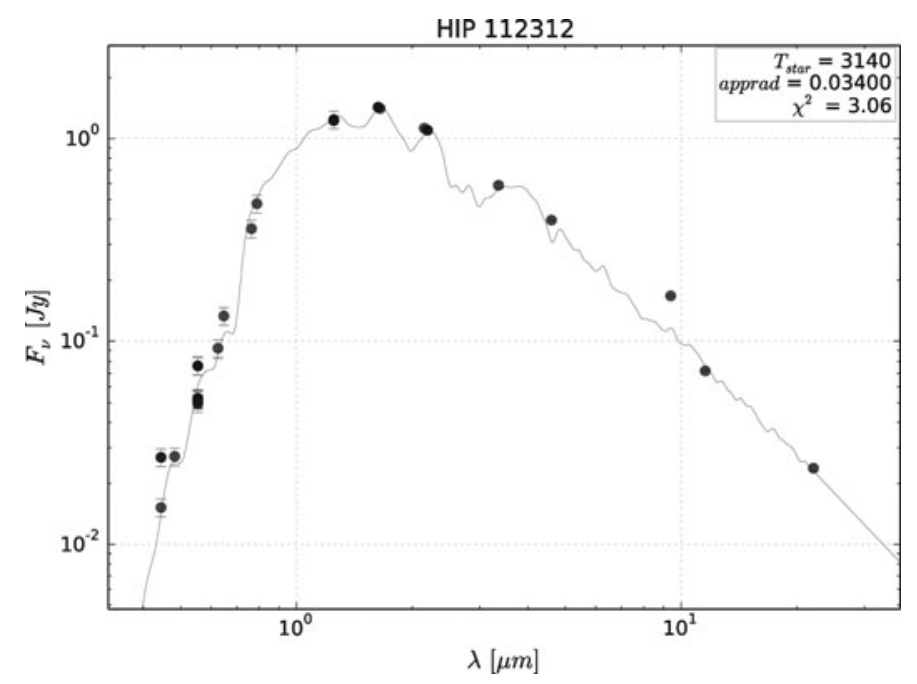

Figure 1. An example of SED fitting, here for BPMG member HIP 112312A.

methods (Song, Zuckerman, \& Bessel 2003; Ortega et al. 2002; Zuckerman \& Song 2004), while older BPMG ages, based on the LDB technique, have been reported more recently (Binks \& Jeffries 2014; Mamajek \& Bell 2014; Malo et al. 2014).

However, as shown in Song et al. (2002), Li depletion calculations from various PMS models are inconsistent. For a given PMS model, there exists a significant discrepancy between the observed LDB luminosity and the expected luminosity. Also, various processes commonly seen among young stars, such as accretion, magnetic fields, and starspots, can affect surface Li depletion. In this paper, I will re-evaluate the LDB age of BPMG using $T_{\text {eff }}$ and luminosity values obtained from spectral energy distribution (SED) fitting, so as to demonstrate the inconsistencies among PMS model calculations.

\section{The LDB Age of the $\beta$ Pictoris Moving Group}

The location of the LDB in terms of temperature and luminosity (hence mass) is a sensitive function of age, so precise measurement of the LDB is important. Observationally, an LDB position is measured in terms of photometric magnitudes and/or colors. Therefore, transforming these observational parameters into temperature and luminosity is necessary, and this has frequently been done using a temperature scale for young stars (e.g., Pecaut \& Mamajek 2013). In this paper, an SED-fitting approach is used instead and, because of the covariance nature of several photometric magnitudes for a given source, this method is known to be very robust (Kraus et al. 2014). For known BPMG members, I have collected 7 or more photometric measurements for each star and these measurements were fit against a set of NextGen synthetic stellar spectra (see Rhee et al. 2007 for details on the SED fit). Best fit parameters ( $T_{\text {eff }}$ and $\theta_{a p p}$, where $\theta_{a p p}$ is the apparent stellar radius in arcseconds) are translated to $T_{\text {eff }}$ and luminosity $(L)$ using the trigonometric parallax distance for each star.

In Figure 1, an example SED fit is displayed; best fit parameters were obtained for other BPMG stars using SED fits of similar quality. Then, estimated temperatures and luminosities were plotted against a set of commonly used pre-main sequence evolutionary models (Figure 2). As shown in Figure 2, the position of the LDB for the BPMG is estimated to be around $T_{\text {eff }}=3050 \mathrm{~K}$ and $L=0.033 L_{\odot}$. This LDB is interpreted as the 


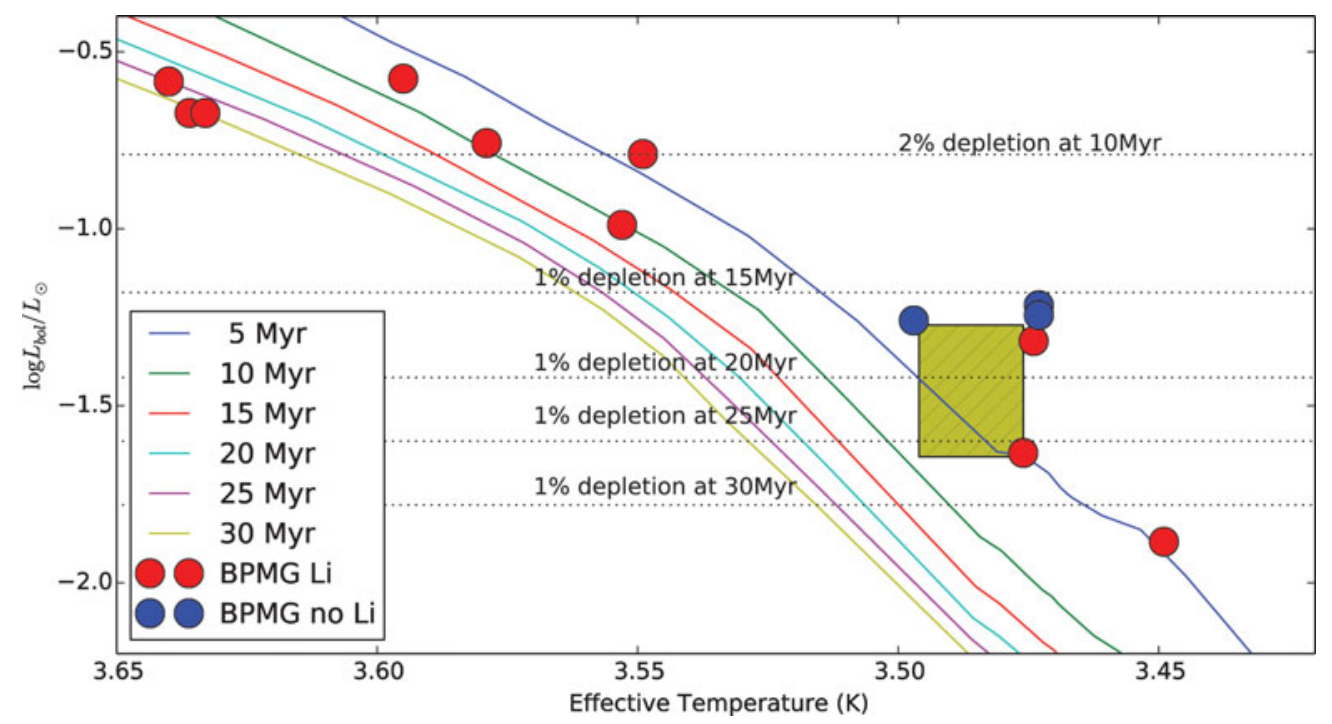

Figure 2. BPMG LDB compared to model lithium depletion for various ages. Model calculations are from Baraffe et al. (2015).

depletion of $\mathrm{Li}$ by more than a factor 100 relative to its initial abundance. In the same figure, several horizontal lines are plotted indicating a factor 100 depletion of lithium for various ages. For PMS models that are consistent, the LDB should appear where a $1 \%$ depletion horizontal line intersects with an appropriate age isochrone. However, the shaded box denoting the observed LDB for the BPMG is not consistent with the lithium depletion pattern, because it is located at a $10 \mathrm{Myr}$ or younger isochrone, where the maximum lithium depletion cannot reach the $100 \times$ level.

Even for fully convective PMS stars (i.e., all stars around the LDB point), temperature is very sensitive to the degree of superadiabadicity in the interior, which in turn depends on the treatment of the convective temperature gradient (Siess et al. 2000). For such cases, an LDB analysis using luminosity has been regarded as more trustworthy than an analysis using temperature (Burrows et al. 2001). Therefore, in LDB analyses, generally only the luminosity is considered. As evident in the Figure, the observed location of the LDB luminosity and the predicted LDB luminosity are very different.

In Song et al. (2002), we used the M4.5+M4.5 BPMG binary system HIP 112312 A\&B, for which a LDB was detected between the two binary components, to illustrate such a PMS model discrepancy, and we advised caution in interpreting the LDB. After ten years, although several more BPMG members have been added, the BPMG LDB is still essentially defined by this same binary system. As shown in the next section, because surface lithium abundance can be affected by stellar evolutionary histories, the LDB detected in a binary should be regarded as more trustworthy than an LDB defined by members of moving groups or clusters. Open cluster (or moving group) members might have been exposed to more diverse evolutionary environments than the two components in a single binary.

\section{Physical Processes Affecting Lithium Depletion in PMS Stars}

It has been shown that a star's previous accretion history can affect its surface lithium abundance at later stage (Baraffe et al. 2002). Also, starspots and magnetic fields can also affect the surface lithium content. 
A high accretion rate results in a smaller stellar radius compared to a non-accreting object, which in turn leads to a hotter core temperature (Baraffe \& Chabrier 2010). Because of the hotter core temperature, lithium can be depleted at a faster rate, in good agreement with the results shown in our LDB analysis.

On the contrary, starspots impact internal pressure and temperature, inhibiting convection. If this effect is significant, then all LDB ages are younger than they should be (Somers \& Pinsonneault 2015). Likewise, the presence of a strong magnetic field in the evolution of a low mass PMS star makes the star appear brighter at a given age (Malo et al. 2014). This effect mitigates the aforementioned apparent discrepancy in the PMS model calculations for lithium depletion.

Because several physical processes can affect surface lithium abundance in a complex manner, it is important to consider all of these effects simultaneously and self-consistently.

\section{Conclusions}

Recently, older ( $\gtrsim 20 \mathrm{Myr}$ ) ages for the BPMG that are based on various LDB analyses have become widely accepted, because of a perception that the LDB age-dating method is based on simpler physics and the fact that multiple research groups have reported similarly old LDB ages for the BPMG. However, as shown earlier in Song, Bessel, \& Zuckerman (2002) and in this paper, there are significant model inconsistencies in PMS calculations. The observed luminosity of the BPMG LDB is different from the expected luminosity obtained from the same model and the inferred LDB age. Until we have self-consistent PMS model calculations, LDB ages have to viewed with caution. Furthermore, there are several important physical processes (accretion, magnetic fields, and starspots) that can change the surface lithium abundance in complex ways. Considering these problems, a new BPMG age of $15 \pm 5 \mathrm{Myr}$ - which spans the previous younger, chromospheric ages and the recent older, LDB ages - is suggested to be used, until a community consensus can be reached.

\section{References}

Baraffe, I., Chabrier, G., Allard, F., \& Hauschildt, P. H. 2002, A\&A A, 382, 563

Baraffe, I. \& Chabrier, G. 2010, A\& A, 521, A44

Baraffe, I., Homeier, D., Allard, F., \& Chabrier, G. 2015, A\& A, 577, A42

Binks, A. S. \& Jeffries, R. D. 2014, MNRAS, 438, L11

Burrows, A., Hubbard, W. B., Lunine, J. I., \& Liebert, J. 2001, RvMP, 73, 719

Kraus, A. L., Shkolnik, E. L., Allers, K. N., \& Liu, M. C. 2014, AJ, 147, 146

Malo, L., Doyon, R., Feiden, G. A., et al. 2014, ApJ, 792, 37

Mamajek, E. E. \& Bell, C. P. M. 2014, MNRAS, 445, 2169

Ortega, V. G., de la Reza, R., Jilinski, E., \& Bazzanella, B. 2002, ApJ, 575, L75

Pecaut, M. J. \& Mamajek, E. E. 2013, ApJS, 208, 9

Rhee, J. H., Song, I., Zuckerman, B., \& McElwain, M. 2007, ApJ, 660, 1556

Siess, L., Dufour, E., \& Forestini, M. 2000, A\&A, 358, 593

Somers, G. \& Pinsonneault, M. H. 2015, MNRAS, 449, 4131

Song, I., Zuckerman, B., \& Bessell, M. S. 2003, ApJ, 599, 342

Song, I., Bessell, M. S., \& Zuckerman, B. 2002, ApJ, 581, L43

Zuckerman, B. \& Song, I. 2004, ARA\&A, 42, 685

\section{Discussion}

ZUCKERMAN: What about LDB ages for other moving groups and open clusters? Would the discrepancy equally affect these other LDB ages?

Author: Yes. The discrepancy will affect all other LDB ages in the same manner. 